\title{
Visual Patterns in Reading Tasks: An Eye-Tracking Analysis of Meares-Irlen Syndrome Simulation Effects
}

\author{
João Vitor Macedo Romera \\ Electrical engineering department \\ Centro Universitário FEI \\ São Bernardo do Campo, SP, Brazil \\ Email: uniejromera@fei.edu.br
}

\author{
Rafael Nobre Orsi \\ Electrical engineering department \\ Centro Universitário FEI \\ São Bernardo do Campo, SP, Brazil \\ Email: rafaelorsi@fei.edu.br
}

\author{
Rodrigo Filev Maia \\ Computer science department \\ Centro Universitário FEI \\ São Bernardo do Campo, SP, Brazil \\ Email: rfilev@fei.edu.br
}

\author{
Carlos Eduardo Thomaz \\ Electrical engineering department \\ Centro Universitário FEI \\ São Bernardo do Campo, SP, Brazil \\ Email: cet@fei.edu.br
}

\begin{abstract}
This work investigates reading patterns based on effects of the Meares-Irlen Syndrome (SMI), a visual-perception deficit that affects indirectly our cognitive system. The most common symptoms related to SMI in reading tasks are visual stress, sensation of moving letters and distortions in the text. These effects have been computationally simulated here and using eye-tracking information of a number of participants we have been able to linearly classify each effects with high accuracy.

Index Terms-Meares-Irlen Syndrome, Eye-Tracking, Reading Patterns.
\end{abstract}

\section{INTRODUÇÃo}

A leitura, que para muitos é uma habilidade natural e automática, é uma das tarefas mais difíceis que o ser humano já desenvolveu ao decorrer de sua evolução, requerendo atividades fisiológicas e cognitivas extremamente complexas. Desde a captação de uma informação pela retina, várias etapas de processos neurológicos devem estar em perfeita sintonia para ser feito o reconhecimento das palavras que estão sendo lidas e associá-las com seu significado e som. Estes processos não são realizados em apenas uma área do cérebro, possibilitando que o estudo da leitura possa ser realizado de várias formas, cognitiva, visual e fonoaudiológicamente [1].

Dentro destes processos, existe a possibilidade de uma das etapas possuir algum tipo de déficit, causado por anormalidades fisiológicas ou cognitivas. Estes déficits podem causar problemas no processo de leitura como um todo, dificultando a compreensão do que está sendo lido, gerando problemas de caráter sociocultural e pedagógico. Estima-se que aproximadamente $17 \%$ da população mundial possui problemas de leitura [2], e, excluindo os casos de dificuldades pedagógicas (condições socioculturais e problemas de ensino) e transtornos neuropsiquiátricos (paralisia cerebral, epilepsia), estimase que a maioria destes casos esteja associado a transtornos neurológicos como a Dislexia ou TDAH [1], [3].
A Dislexia e o TDAH são os distúrbios mais presentes no ambiente escolar, interferindo principalmente no rendimento, deixando-o muito abaixo do esperado em relação à idade do indivíduo portador [4]. No caso da Dislexia, o indivíduo não possui déficits perceptuais e mesmo assim apresenta dificuldade na leitura. Segundo a literatura [3]-[5], existem três tipos distintos de classificação para a Dislexia: fonológica, lexical ou mista. Em todos os tipos, o problema está centrado no processo cognitivo da leitura, ou seja, é um problema exclusivamente neurológico. Os sintomas levam a problemas que muitas vezes são identificados como desatenção na hora da leitura, desinteresse e perda de autoestima (principalmente quando o portador está no processo de aprendizagem primário) [3]. Já no caso do TDAH (Transtorno de Déficit de Atenção/Hiperatividade), o indivíduo também possui dificuldade no processo de leitura, sendo caracterizado pela frequente perda de atenção e hiperatividade/impulsividade [5].

Dentre os distúrbios da capacidade de leitura, existe a possibilidade do déficit estar presente em uma capacidade visual-perceptiva, relacionado a uma deficiência na retina que causa uma desordem no processamento das informações captadas [2], denominada Síndrome de Meares-Irlen (SMI) [6]. A SMI pode vir acompanhada de outros distúrbios, o que dificulta seu diagnóstico, que muitas vezes é deixado de lado por possuir sintomas muito próximos à Dislexia e ao TDAH. Dentre os principais sintomas da SMI estão presentes a dificuldade de leitura e estresse visual, porém estas condições não são causadas por fatores neurológicos, apesar do processo cognitivo da leitura ficar comprometido e ser prejudicado.

Os exames oftalmológicos tradicionais não conseguem detectar essa síndrome, requerendo o desenvolvimento de novos métodos para obter um diagnóstico preciso. Estudos recentes [1], [2] pesquisam como quantificar dados relacionados à visão para que possam ser comparados com dados obtidos de 
indivíduos com suspeitas de portar a síndrome, na intenção de detectar as características exclusivas dos portadores da síndrome de Irlen. Com estas características identificadas, é possível realizar exames para a detecção da síndrome. O método mais utilizado hoje [2] possui caráter neuropsiquiátrico, dependendo de um profissional da área para analisar o desempenho da leitura do indivíduo. Por partir de uma análise subjetiva, o diagnóstico fica impreciso, sugerindo o desenvolvimento de um novo método, utilizando tecnologias que permitem obter dados que antes eram impossíveis de ser mensurados.

O objetivo deste artigo é realizar uma investigação menos subjetiva sobre as diferenças entre os padrões visuais de leitura em distorções (ou efeitos) comumente relacionadas à SMI, como embaçamento das letras, sensação de letras em movimento e espaçamento irregular entre as palavras, geradas sinteticamente em imagens de texto literários. Mais especificamente, este artigo (1) desenvolve um algoritmo para gerar estímulos artificiais que simulam computacionalmente distorções visuais percebidas por pessoas portadoras da SMI, (2) realiza um experimento com eye-tracking utilizando essas distorções visuais simuladas com o propósito de coletar sinal visual correspondente, (3) cria uma base de dados composta de imagens de padrões visuais de leitura extraídos do eyetracker em textos com tais distorções, e (4) implementa um arcabouço computacional tradicional, mas inédito para reconhecer padrões visuais de leitura automaticamente.

\section{SÍnDROME DE MEARES-IRLEN}

A SMI é uma deficiência na capacidade visual-perceptiva humana que causa a desorganização das informações captadas pelos olhos e distorções no processamento destas informações pelo córtex visual [7]. Essa deficiência prejudica várias habilidades humanas dependentes da visão, tais como ler, escrever, dirigir veículos e praticar esportes.

Dentre essas habilidades, a mais afetada e explorada atualmente é a de leitura, que se torna lenta e descontínua, dificultando a compreensão do texto lido. Indivíduos portadores de SMI relatam cansaço visual, dor nos olhos, lacrimejamento e estresse visual após períodos de no máximo 15 minutos de esforço [6].

As principais manifestações da SMI no processo de leitura estão associadas a alta sensibilidade à luz, provocando distorções devido ao contraste branco/preto de impressões em papel sulfite, embaçamento das letras, sensação de letras em movimento e espaçamento irregular entre as palavras [1], [3].

Estima-se que a SMI afete aproximadamente $15 \%$ da população mundial, e que $45 \%$ das pessoas que possuem dificuldades de leitura possuem diagnóstico positivo para essa síndrome [2]. No entanto, por possuir sintomas similares a outros transtornos relacionados à leitura e, muitas vezes, vir acompanhada destes transtornos, o diagnóstico da SMI se torna uma tarefa difícil, pois é realizado analisando-se fundamentalmente o processo de leitura do indivíduo.

Neste contexto, estudos recentes [1], [2] sobre a SMI que fazem uso de equipamento de rastreamento de olhar (em inglês, eye-tracking) têm revelado achados inéditos que permitem uma análise mais específica das pessoas portadoras da síndrome. Basicamente, o uso do equipamento de rastreamento de olhar durante o processo de leitura de um indivíduo permite investigar o movimento e a dilatação da pupila do voluntário, qualificando e quantificando métricas como o número de regressões (movimentos da direita para a esquerda), fixações (fixações do olhar da esquerda para a direita), a relação de palavras lidas por minuto durante a leitura, a tendência de se ler da esquerda para a direita, o alcance do reconhecimento (número de palavras lidas por fixação), entre outras [2].

Entretanto, as métricas atuais, no estágio em que se encontram, não descrevem informações discriminantes sobre padrões de leitura impactados por distorções distintas no processamento visual destas informações, trazendo resultados cientificamente questionáveis que não têm sido reconhecidos como válidos por várias organizações, tais como a Academia Americana de Pediatria e a Sociedade Brasileira de Oftalmologia Pediátrica [8].

\section{MATERIAIS E MÉtodos}

Esta seção apresenta o conjunto de materiais e métodos utilizados na realização do experimento e está dividida em cinco subseções: estímulo visual; participantes; aquisição do sinal; pré-processamento e reconhecimento de padrões.

\section{A. Estímulo visual}

A partir de exemplos de distorções visuais [9] que uma pessoa diagnosticada com a SMI supostamente visualiza ao ler um texto estático [6], foi desenvolvido um programa para simulação de efeitos visuais dinâmicos a partir de textos e parâmetros inseridos pelo usuário.

Os efeitos simulados foram produzidos utilizando as bibliotecas de manipulação de imagens (Magick) e gifs (ggplot2) da plataforma de processamento de dados $R$ Studio. As funções da biblioteca "Magick" permitem a transformação de textos (que são inseridos pelo usuário) em imagens no formato .jpeg enquanto a biblioteca "ggplot2" organiza as imagens em quadros (ou frames), gerando um vídeo que simula um efeito dinâmico da SMI.

A seguir, descreve-se o pseudocódigo que explica o procedimento utilizado para gerar os efeitos mostrados nas Figuras 1 e 2.

\begin{tabular}{l}
\hline Algorithm 1 Pseudocódigo para geração dos efeitos \\
Entradas: Texto, Tamanho da fonte, Tipo da fonte, Cor da \\
fonte, Tipo de efeito e Tempo de duração \\
Leia: Parâmetros de entrada \\
Inicio \\
Organiza variáveis de configuração \\
Aplica efeito de animação com "ImageMagick" \\
Agrupa imagens com efeito de animação \\
Renderiza vídeo da animação \\
Fim \\
Saída:Texto com efeito dinâmico no formato .avi
\end{tabular}


Maria era uma ovelha. Onde as outras ovelhas iam, Maria ia também. As ovelhas iam pra cima. Maria ia pra cima. Maria ia sempre com as outras. a) Um dia todas as ovelhas foram para o Pólo Sul.

\section{Maria era uma ovelha. Onde as outras ovelhas iam, Maria ia também. As ovelhas iam pra cima. Maria ia pra cima. Maria ia sempre com as outras. b) Um dia todas as ovelhas foram para o Pölo Sul.}

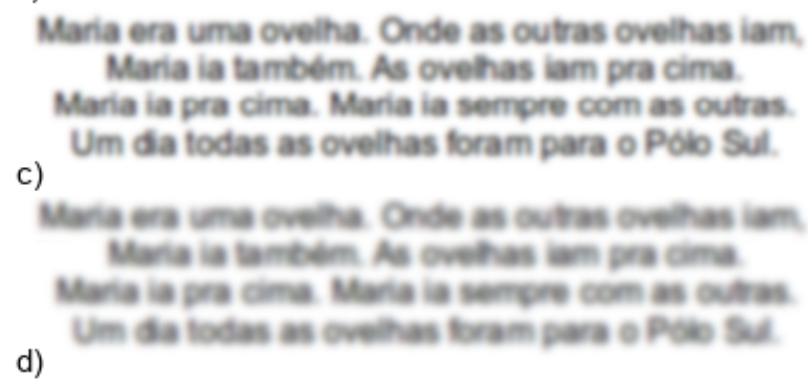

Figura 1. Efeito Blurry codificado: a) Imagem original; b) Blurry com sigma $=1$; ) Blurry com sigma $=1.5$; d) Blurry com sigma $=2$.

\section{Maria era uma ovelha. Onde as outras ovelhas iam, Maria ia também. As ovelhas iam pra cima. Maria ia pra cima. Maria ia sempre com as outras. Um dia todas as ovelhas foram para o Pólo Sul. \\ Maria era una ovettia. Cnde as outras ovel has iam, Maria ia tambem. As ovelhas iam pra cima. Maria ia pra cima. Maria ia sempre com as outras. ) Un dia todas as ovelhas foram para o Pón :ul. \\ Niaria era uma vivelhä. Onde as outras crelhas tam,

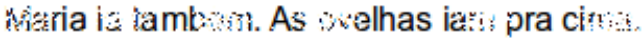

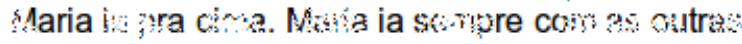 \\ Um dia todas as ovelmos foran para o Pcio Sul.

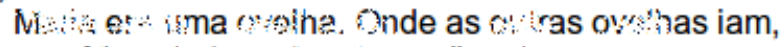

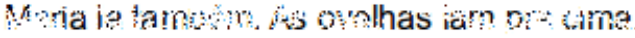

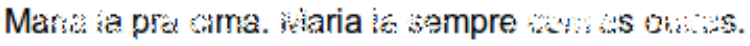

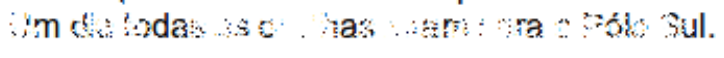
a)

b)

c)$$
\text { d) }
$$

Figura 2. Efeito Washout codificado: a) Imagem original; b) Washout com sigma $=1 ; \mathrm{c}$ ) Washout com sigma $=1.5 ;$ d) Washout com sigma $=2$.

Para cada efeito programado é utilizado um parâmetro (sigma) que indica a intensidade do efeito aplicado (quanto maior o valor de sigma, maior é a intensidade do efeito) e um parâmetro (tau) que indica o tempo de ciclo do efeito. A partir destes parâmetros a distorção visual é alterada dinamicamente, se intensificando e retornando ao texto original gradativamente, como mostra os exemplos nas Figuras 1 e 2 que possuem valores de sigma variando entre zero (texto neutro) e dois (texto com maior distorção).
Neste trabalho, essas distorções visuais simuladas foram utilizadas em um experimento de leitura para registrar os movimentos oculares de indivíduos voluntários que supostamente não possuem a SMI, compondo uma amostra de dados quantificados de regressões, fixações, fluência na leitura e outras variáveis que juntas permitem a concepção de um padrão visual sintético para cada efeito da SMI.

Os estímulos visuais utilizados neste experimento foram desenvolvidos a partir de histórias da literatura infantil, com perguntas literais e inferenciais, que foram apresentadas na tela do equipamento de rastreamento ocular durante as sessões de leitura.

\section{B. Participantes}

O experimento foi realizado com 70 participantes voluntários, sendo 30 homens e 40 mulheres, com idades entre 8 e 52 anos. Todos saudáveis e cognitivamente capazes.

\section{Aquisição do sinal}

A aquisição do sinal foi realizada em ambiente fechado com iluminação artificialmente controlada dentro das especificações ideais entre 300 e 1000lux [10]. Os equipamentos utilizados foram o rastreador de olhar (eye-tracker) Tobii TX300 juntamente com um notebook com processador core i7 e 16 Gb de RAM, um teclado comum (padrão ABNT) e sistema operacional Windows 7, para que fosse utilizado o software Tobii Studio durante as gravações do sinal.

A parte inicial do experimento constituiu nas instruções para a realização do teste juntamente com acomodação do participante em frente ao equipamento de rastreamento ocular. Em seguida é ajustada a posição de cada participante dentro do alcance de medição do equipamento, como é apresentado na Figura 3 e então é realizada a calibração do equipamento.
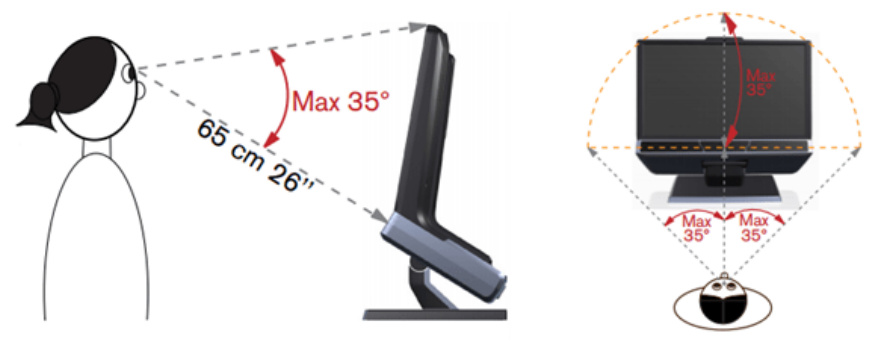

Figura 3. Parâmetros do equipamento eye-tracker para aquisição do sinal.

Posteriormente, foram apresentados três textos distintos no eye-tracker: o primeiro sem efeito (Neutro), o segundo com o efeito borrado (Blurry) e o terceiro com o efeito embaçado (Washout). Ao final de cada texto, uma pergunta era exibida na tela e o participante respondia as perguntas em voz alta, para anotação dos acertos e erros em cada texto para cada voluntário. Os textos possuíam tempo limite de exposição (30 segundos), ao passo que as perguntas não possuíam tempo limite e era requisitado que o participante pressionasse alguma tecla presente em um teclado disposto à sua frente, para que fosse exibido o próximo texto. 
NEUTRO (SEM EFEITO)
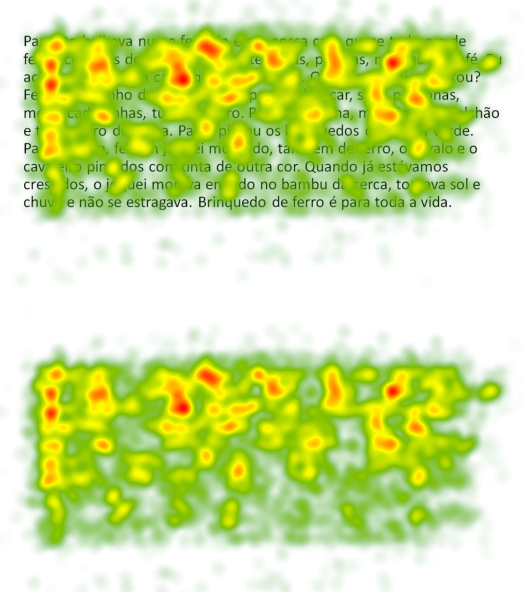

BLURRY (BORRADO)
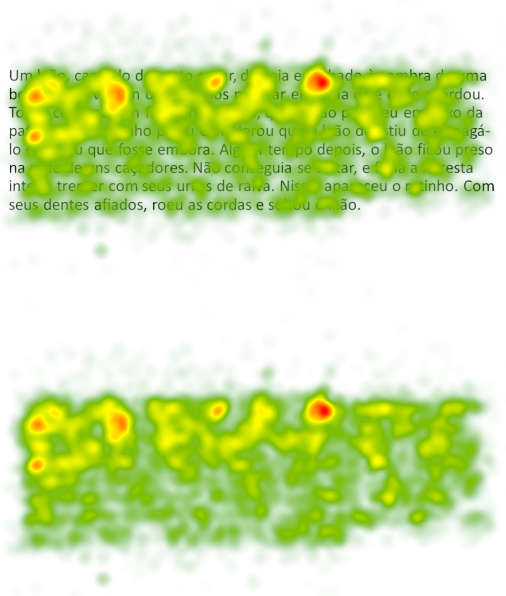

WASHOUT (EMBAÇADO)
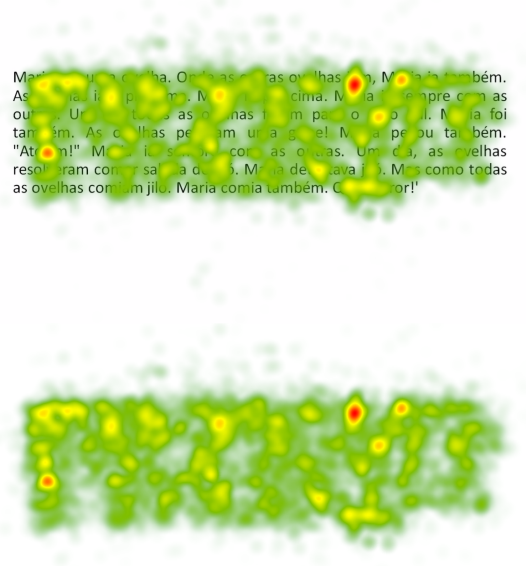
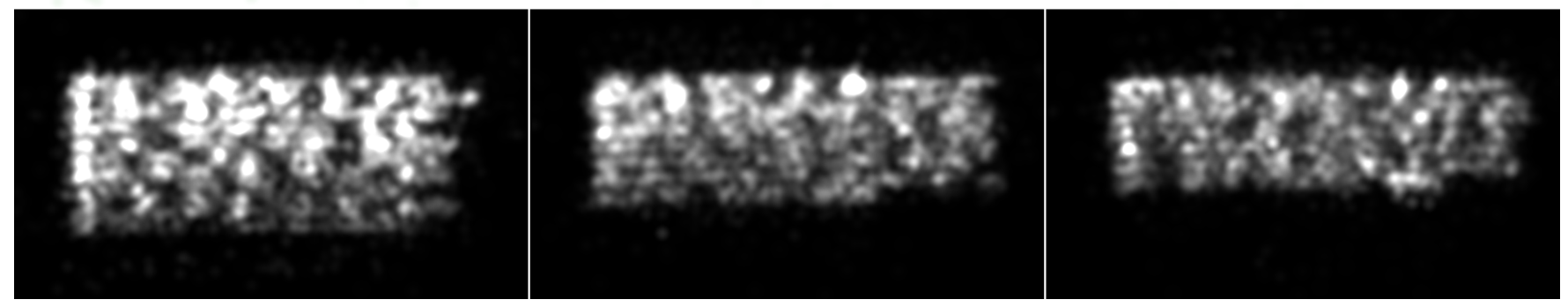

Figura 4. Pré-processamento do sinal de entrada.

\section{Pré-processamento}

A partir do experimento realizado, foram gerados os mapas de atenção visual de cada participante por estímulo, como pode ser visto nos três primeiros quadros da parte superior da Figura 4.

O mapa de atenção visual é obtido a partir da consolidação das fixações oculares em uma formatação gráfica do tipo mapa de calor, o qual leva em consideração duas medidas: 1) o limiar de espaço que indica área máxima em pixels para computar uma fixação e; 2) o limiar de tempo que indica o tempo mínimo para contabilizar cada fixação [11]. Neste trabalho esses limiares foram configurados com área de 40 pixels e tempo de $60 \mathrm{~ms}$, formatando uma máscara de pontos em que os dados de fixação são computados e acumulados para gerar as graduações do mapa de calor, que variam do vermelho (pontos de maior incidência) ao verde (pontos de menor incidência) [12].

$\mathrm{Na}$ etapa de pré-processamento foi feita a separação do fundo da imagem (texto apresentado como estímulo) dos dados de fixação ocular (mapa de atenção visual), em seguida o mapa de calor foi convertido para mapa de opacidade, contendo apenas tons de cinza, conforme é apresentado nos três últimos quadros da Figura 4.

Todas as imagens geradas com o experimento possuem a mesma resolução (1280x720), orientação e intensidade de luminosidade, resultando em uma base de dados normalizada com 210 imagens de padrões visuais de leitura, sendo 70 imagens de cada padrão estudado (Neutro, Blurry e Washout).

\section{E. Reconhecimento de padrões}

$\mathrm{Na}$ etapa de reconhecimento de padrões, nós implementamos um arcabouço computacional baseado em estatística multivariada que utiliza Análise de Componentes Principais (PCA) [13] e Análise de Discriminantes Lineares de Máxima Incerteza (MLDA) [14] para extrair informações discriminantes das imagens [14].

Neste arcabouço, apresentado na Figura 5, o PCA é utilizado para reduzir a dimensionalidade dos dados (item C) [13], [15], [16]. Previamente, no item A, é selecionado o conjunto de dados de entrada de dimensão $N$ x $n$ (que neste trabalho possui dimensão 210x921.600) e é subtraído o seu sinal médio, resultando em um conjunto de dados com média igual a zero (item B). Então é calculada a matriz de transformação linear e é feita a redução da dimensionalidade do sinal para obter a matriz de componentes principais $n \mathrm{x} m$, tal que $m=N-1$, armazenando todos os autovetores não-nulos. A matriz resultante desta operação (agora com dimensão 210x209) é utilizada como entrada para calcular o autovetor discriminante do MLDA (item $D$ ). Por fim (item F), obtém-se uma matriz de projeção $(N \mathrm{x} 1)$ com as características mais discriminantes de cada um dos $N$ vetores de entrada. 


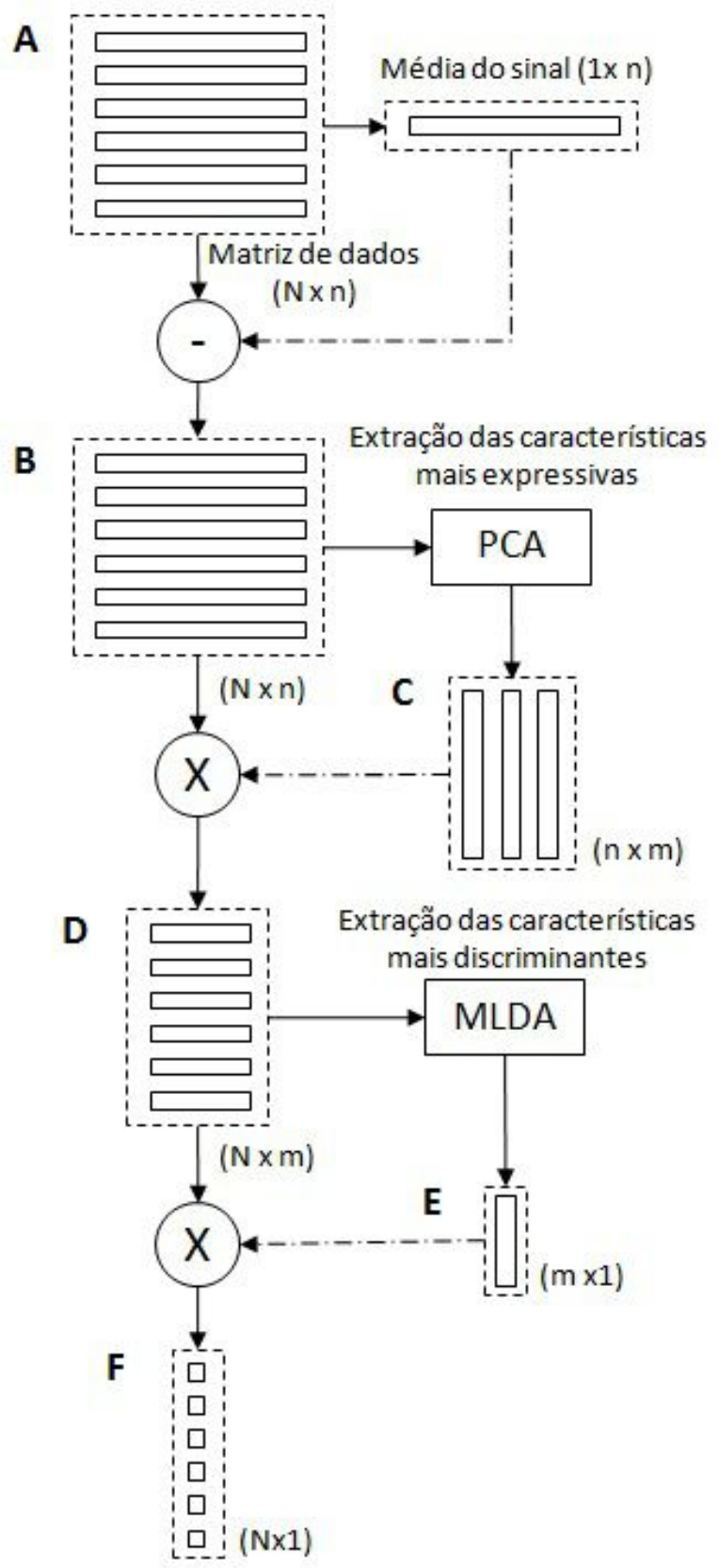

Figura 5. Arcabouço computacional para extração de características e classificação.

\section{REsultados}

Para avaliar o desempenho de classificação do arcabouço computacional proposto, foi adotado o método $k$-fold [17]. Este método consiste em fazer uma validação cruzada dividindo um conjunto de dados em $k$ subconjuntos exclusivos e de mesmo tamanho. A partir desta divisão, um subconjunto é separado para a validação e os demais subconjuntos são utilizados para o treinamento do classificador. Este processo é repetido $k$ vezes, alterando o subconjunto de validação. Ao final das $k$ iterações é obtida a acurácia do classificador apurando os erros encontrados e calculando a média aritmética dos acertos em cada teste. A Figura 6 e a Tabela I apresentam os resultados obtidos.

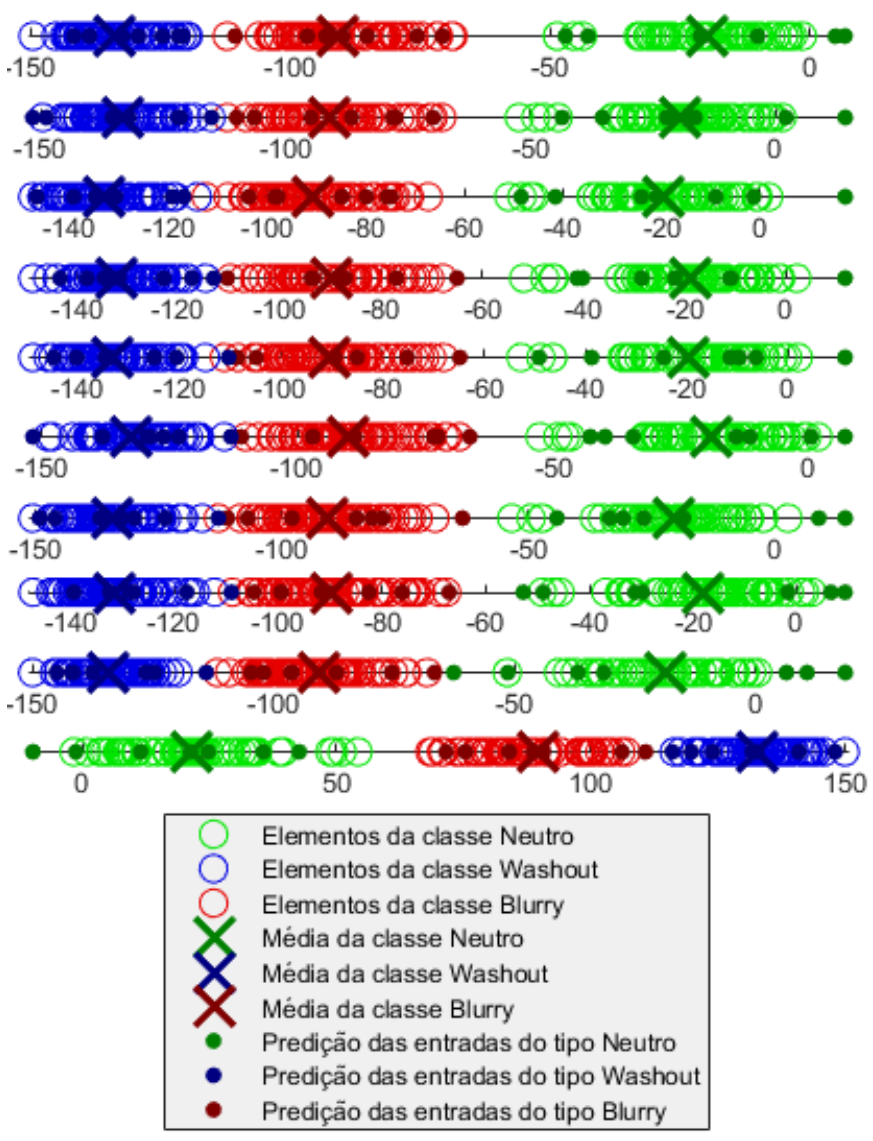

Figura 6. Teste de validação cruzada.

Tabela I

ACURÁCIA DO TESTE DE VALIDAÇÃO CRUZADA

\begin{tabular}{ccccc} 
K & NEUTRO & BLURRY & WASHOUT & $\overline{\mathbf{X}}$ \\
\hline k1 & $100 \%$ & $100 \%$ & $100 \%$ & $100 \%$ \\
k2 & $100 \%$ & $100 \%$ & $100 \%$ & $100 \%$ \\
k3 & $100 \%$ & $100 \%$ & $100 \%$ & $100 \%$ \\
k4 & $100 \%$ & $100 \%$ & $100 \%$ & $100 \%$ \\
k5 & $100 \%$ & $100 \%$ & $85,71 \%$ & $95,24 \%$ \\
k6 & $100 \%$ & $100 \%$ & $100 \%$ & $100 \%$ \\
k7 & $100 \%$ & $100 \%$ & $100 \%$ & $100 \%$ \\
k8 & $100 \%$ & $100 \%$ & $85,71 \%$ & $95,24 \%$ \\
k9 & $85,71 \%$ & $100 \%$ & $100 \%$ & $95,24 \%$ \\
k10 & $100 \%$ & $100 \%$ & $100 \%$ & $100 \%$ \\
\hline \multicolumn{5}{c}{ Acurácia Total } \\
5
\end{tabular}

Como apresentado na Tabela I, obtivemos um elevado índice de acertos com o classificador proposto (98,57\%), foram 207 acertos em 210 testes. O fator predominante para o êxito na tarefa de classificação foi a distribuição dos dados no hiperplano discriminante, pois, como pode ser visto na Figura 7, praticamente não houve sobreposição dos dados na maioria dos testes realizados, o que propicia uma boa separabilidade linear das classes e, consequentemente, uma boa precisão na projeção dos dados de validação. 

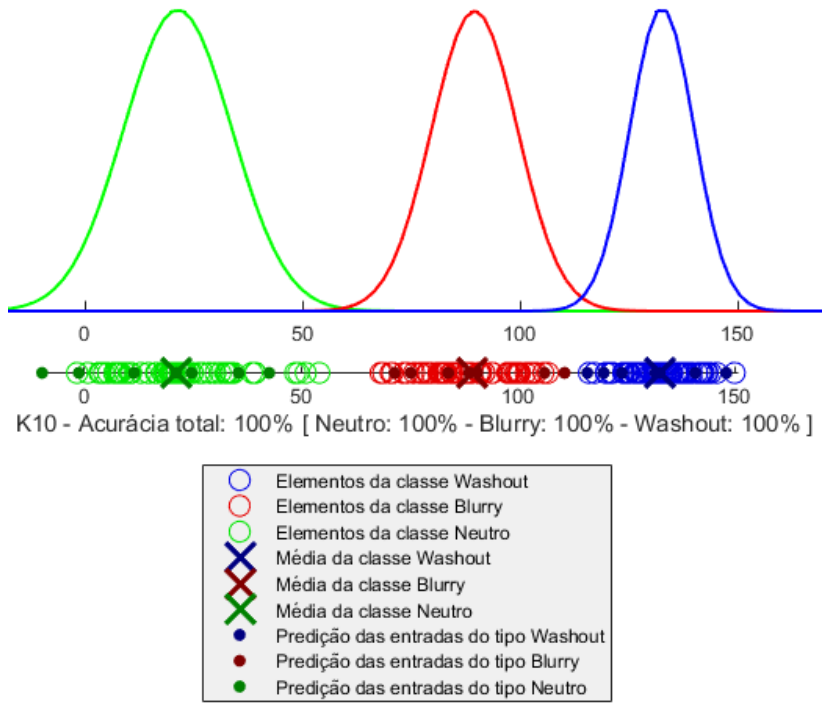

Figura 7. Exemplo de separação das classes em um dos testes de validação do classificador (teste k10).

Também pudemos constatar que a diferença entre as imagens é estatisticamente significativa, com $p$-value menor que $5 \%$ em todas as comparações.

1) Neutro $X$ Blurry: $p$-value $=6.3458 \mathrm{e}-65$

2) Neutro $X$ Washout: $p$-value $=1.5456 \mathrm{e}-94$

3) Blurry X Washout: $p$-value $=4.5323 \mathrm{e}-55$

\section{Conclusẽo}

Embora haja uma estimativa de que $15 \%$ da população mundial possua diagnóstico positivo para a SMI [2], ainda existem poucos trabalhos na literatura sobre esse assunto. $\mathrm{Na}$ verdade, ainda não se sabe se a SMI é uma deficiência que está associada às células receptoras dos olhos ou ao processamento cognitivo de interpretação das imagens. Ambas as hipóteses nos levam a problemas complexos devido a robustez dos órgãos envolvidos, pois aproximadamente $40 \%$ do córtex cerebral e cerca de $70 \%$ de todos os receptores sensitivos do corpo humano (presentes nos olhos) estão diretamente relacionados ao sentido da visão [18].

Os resultados experimentais deste artigo indicam que a extração e classificação linear de padrões visuais de leitura por meio de rastreamento ocular e técnicas de estatística multivariada são métodos promissores para avaliar objetivamente como uma pessoa interpreta um texto literário e, consequentemente, correlacionar esse esforço cognitivo com distorções visuais percebidas por aqueles que possuem SMI.

Todos os algoritmos descritos aqui serão implementados futuramente em uma plataforma de processamento na nuvem (shiny.rstudio.com) e disponibilizados para a comunidade científica, permitindo que outros pesquisadores possam gerar efeitos que simulem a SMI a partir de seus próprios textos, contribuindo para o desenvolvimento de soluções científicas e tecnológicas afins.

\section{AgRadecimentos}

Os autores deste trabalho gostariam de agradecer o apoio da FEI (PBIC: 152/18) e da CAPES (código de financiamento 001). Adicionalmente os autores gostariam de agradecer a todos os voluntários participantes do experimento.

\section{REFERÊNCIAS}

[1] M. R. Guimaraes and R. Guimaraes, "Por que ler pode ser tão difícil síndrome de irlen \& distúrbios do processamento visual pela via magnocelular," XXVI Encontro Nacional de Professores do PROEPRE, 2013.

[2] L. F. Bicalho, M. Z. T. de ALMEIDA, M. R. Guimarães, J. R. G. Silva, and F. Fully, "Síndrome de irlen: Um olhar atendo sobre o funcionamento cerebral durante a leitur," Acta Biomedica Brasiliensia, vol. 6 , no. 1 , pp. 35-44, 2015.

[3] L. N. de Faria, "Frequência da sindrome de meares-irlen entre alunos com dificuldades de leitura observadas no contexto escolar," Instituto de Ciências Biológicas da UFMG, 2011.

[4] S. A. Capellini, T. d. L. Ferreira, C. A. Salgado, S. M. Ciasca et al. "Desempenho de escolares bons leitores, com dislexia e com transtorno do déficit de atenção e hiperatividade em nomeação automática rápida," Revista da Sociedade Brasileira de Fonoaudiologia, 2007.

[5] A. H. d. Amaral, M. M. Guerreiro et al., "Transtorno do déficit de atenção e hiperatividade: proposta de avaliação neuropsicológica para diagnóstico," Arquivos de Neuro-Psiquiatria, 2001.

[6] H. L. Irlen, "Method and apparatus of treatment of symptoms of the irlen syndrom," Oct. 9 1990, uS Patent 4,961,640.

[7] F. A. Soares and L. S. Gontijo, "Production of knowledge: genetic basis, biochemical and immunological of meares-irlen syndrome," Revista Brasileira de Oftalmologia, vol. 75, no. 5, pp. 412-415, 2016.

[8] P. D. M. B. Tartarella, S. D. G. C. Vasconcelos, and T. D. F. Ejzenbaum, "Parecer da sociedade brasileira de oftalmologia pediátrica (sbop) sobre síndrome de irlen e a falta de evidência científica que recomende o uso de lentes ou filtros coloridos para essa situação," São Paulo/Brasil, 2017.

[9] IrlenClinic, "For reading and perceptual disorders and dyslexia diagnosis," 2017, accessado em 08-08-2019. [Online]. Available: http://irlenclinic.com.au/irlensymptoms/distortions/

[10] Accuracy and precision test report, 2nd ed., Tobii Technology, 2013, rev $\mathrm{AB}$.

[11] A. T. Duchowski, Eye tracking methodology. Springer, 2017.

[12] L. Silva, "Um estudo sobre mapeamento cerebral e análise de movimentos oculares em jogadores de xadrez," Master's thesis, Centro Universitário FEI, São Bernardo do Campo - SP, Brazil, 2 2017, 65.f. Dissertação (Mestrado em Engenharia Elétrica) - Centro Universitário FEI, São Bernardo do Campo, 2017.

[13] K. Fukunaga, Introduction to statistical pattern recognition, 2nd ed. Academic press, 2013.

[14] C. E. Thomaz, J. P. Boardman, S. Counsell, D. L. Hill, J. V. Hajnal, A. D. Edwards, M. A. Rutherford, D. F. Gillies, and D. Rueckert, "A multivariate statistical analysis of the developing human brain in preterm infants," Image and Vision Computing, vol. 25, no. 6, pp. 981-994, 2007.

[15] H. Abdi and L. J. Williams, "Principal component analysis," Wiley interdisciplinary reviews: computational statistics, vol. 2, no. 4, pp. 433459, 2010.

[16] E. Z. Tenorio and C. E. Thomaz, "Anàlise multilinear discriminante de formas frontais de imagens $2 \mathrm{~d}$ de face," in Proceedings of the X Simposio Brasileiro de Automacao Inteligente SBAI, 2011, pp. 266-271.

[17] Y. Bengio and Y. Grandvalet, "No unbiased estimator of the variance of k-fold cross-validation," Journal of machine learning research, vol. 5, no. Sep, pp. 1089-1105, 2004.

[18] E. N. Marieb, P. B. Wilhelm, and J. Mallat, Anatomia humana, 7th ed. São Paulo: Pearson Education do Brasil, 2014. 